\title{
Bioética clínica: contribuições para a tomada de decisões em unidades de terapia intensiva neonatais
}

\author{
Clinical bioethics: contributions to decision-making \\ in neonatal intensive care units
}

Carlos Dimas M artins Ribeiro ${ }^{1}$

Sergio Rego ${ }^{2}$

${ }^{1}$ Instituto deSaúdeda

Comunidade, Universidade

Federal Fluminense. Rua

M arquês do Paraná 303,

segundo andar do anexo do

H ospital Antônio Pedro,

Centro. 24033-900

Niterói RJ.

dimasribeiro@cremerj.org.br

${ }^{2}$ Escola N acional deSaúde

Pública, Fiocruz.
Abstract This article presents a reflection about decision-making in N eonatal IntensiveCareU nits based on the principle of distributive justice and considering the theoretical approaches of the Bioethics of Protection, formulated by Schramm and Kottow, and the Theory of Capabilities developed by N ussbaum and Sen. Within the concept of justice in the health area adopted in this study, we characterize the essential needs of the citizens that must besatisfied by theState. Then we discuss the question of who should be benefited in the neonatal intensive care units- and in which way - given the scarce public resources available, considering three groups of newborn that in general represent the demand for intensive care services in these units. We conclude that, the better the clinical information available, the more it is likely that a better and more qualified ethical choice can be taken. Further studies will be necessary for the construction of reasonable evidence, prognosisincluded, so that the involved families can be provided with even more adequate information and, most importantly, for allowing the parents of the child to take part in the clinical decisions. Key words Bioethics of protection, Theory of capabilities, Justice in health, Clinical bioethics, Neonatal ICU
Resumo 0 artigo oferece uma reflexão sobre a tomada de decisão nas UTI neonatais levando-se em conta o princípio da justiça distributiva, tendo como referenciais teóricos complementares a bioética de proteção, elaborada por Schramm e Kottow, ea teoria das capacidades ("capability"), formulada por Nussbaum e Sen. Em um primeiro momento, apresenta-se a abordagem das capacidades e a bioética de proteção, caracterizando-se, dentro da concepção de justiça sanitária adotada neste trabalho, quais são as necessidades essenciais dos cidadãos queo Estado tem o dever de satisfazer para que haja justiça. Em seguida, discute-se a questão de quem deve ser ben eficiado na UTI neonatal - e de que maneira - com os recursos públicos disponíveis, considerando-se três grupos de re cém-nascidos que, de forma geral, compõem a demanda pelo atendimento nestes serviços. Concluise que a decisão ética será também mais qualificada quanto melhor for a informação clínica que estiver disponível, sendo necessário desenvolver mais estudos que amparem a construção de evidências razoáveis, inclusive relativas ao prognóstico, para que se possa esclarecer de forma ainda mais apropriada às famílias envolvidas. E, especialmente, a importância de envolver os pais da criança na tomada de decisões clínicas.

Palavras-chave Bioética de proteção, Teoria das capacidades, Justiça sanitária, Bioética clínica e UTI neonatal 
Introdução

Neste trabalho, discutiremos a tomada de decisão nas Unidades de Terapia Intensiva (UTI) neonatais do ponto de vista da justiça distributiva. Esta se refere à justa distribuição das cargas riscos, custos, danos - e dos benefícios - bens, serviços, tecnologias - resultantes da cooperação entre os cidadãos que compõem a sociedade. Estas cargas e benefícios são distribuídos através de um conjunto de instituições econômicas, políticas e sociais que compõem a estrutura básica de uma dada sociedade, mantendo o Estado, nas sociedades contemporâneas, um papel fundamental na sua distribuição ${ }^{1}$. Desta forma, a justiça distributiva trata da relação entre governantes e governados, avaliando-se em que medida as políticas públicas promovem uma distribuição justa das cargas e benefícios.

Duas condições são necessárias para que problemas de justiça sejam reconhecidos ${ }^{1}$. A primeira éa escassez de recursos - condição objetiva -, e a segunda é o pluralismo moral - condição subjetiva, ambas presentes nas sociedades democráticas contemporâneas, como é, em princípio, a brasileira. Se os recursos existentes fossem abundantes a ponto de satisfazer todos os desejos e necessidades das pessoas, e se todas as pessoas concordassem sobre as prioridades na alocação dos mesmos, não existiriam questões de justiça.

A condição subjetiva pode ser caracterizada como a vigência, em sociedades democráticas, de uma diversidade de comunidades morais, com diferentes doutrinas capazes de fornecer uma orientação ética aos cidadãos, que, em muitas situações, são incompatíveis entre si ${ }^{2,3}$. Assim, os indivíduos e os grupos no interior destas sociedades muitas vezes divergem, em vista de seus interesses e valores, sobre a questão de queserviços devem ser oferecidos com recursos sanitários públicos e para quem.

N este artigo, refletiremos sobre a justiça sanitária, tendo como pressuposto o dever do Estado de satisfazer as necessidades essenciais de seus cidadãos, distribuindo de forma justa os recursos públicos. No caso brasileiro, esta obrigação do Estado está prevista na atual constituição brasileira, na qual a saúde é entendida como qualidade de vida e como um bem público que deve ser garantido pelo Estado como um direito de todos os cidadãos. Para tanto, será focalizado, como tema específico, a alocação de recursos para a atenção intensiva neonatal.

As UTIs neonatais são estruturas assistenciais que objetivam a atenção aos recém-nasci- dos extremamente doentes - cuja condição clínica constitui uma ameaça imediata ou potencial à vida -, que requerem interven ções com tecnologias médicas sofisticadas e caras, como nos casos de prematuridade e enfermidades congênitas ${ }^{4}$.

$\mathrm{Na}$ análise aqui empreendida, foram utilizadas como ferramentas teóricas a teoria das capacidades formulada por Nussbaum ${ }^{5,6}$ e $\operatorname{Sen}^{7,8}$ e a bioética de proteção proposta por Schramm e Kottow ${ }^{9}$ e Kottow ${ }^{10}$. Estas ferramentas teóricas nos permitem caracterizar, dentro da concepção de justiça distributiva e de justiça sanitária que estamos adotando neste trabal ho, o que se pretende igualar para que a distribuição das cargas e ben efícios possa ser considerada justa. Em outras palavras, diante da heterogeneidade básica dos seres humanos - diferentes em suas condições sociais, suas particularidades corporais, seus talentos e suas concepções do bem, entre outros componentes - qual seria o espaço apropriado para avaliar a igualdade - ou desigualdade - entre os indivíduos e os grupos sociais, de modo a poder dizer que uma dada sociedade é justa ou injusta ${ }^{8}$ ? 0 que é devido a cada um cidadão para que haja justiça ou quais são as necessidades essenciais dos cidadãos que o Estado tem o dever de satisfazer eporque estas necessi dades são essenciais em oposição a outras necessidades que não 0 são. Em relação ao tema específico que estamos discutindo aqui, a pergunta é o que é devido a cada um na UTI neonatal, considerando-sea justa alocação dos recursos sanitários públicos?

Proteção, capacidades e dignidade humana

Para responder o que é devido a cada cidadão, comecemos com uma distinção, proposta pela bioética de proteção, entre vulnerabilidade primária ou existencial evulneração. A primeira diz respeito à condição universal da espéci e humana e, mutatis mutandis, de qualquer ser vivo, que são seres suscetíveis de sofrer danos ${ }^{10,11}$. A vulneração refere-se à vulnerabilidade secundária ou circunstancial a que estão sujeitos certos seres humanos por viverem em condições econômicas esociais particulares de privação de suas necessidades essenciais ${ }^{12}$.

Para a bioética de proteção, o requerimento ético diante dessas duas formas de vulnerabilidade é a proteção, entendida como a responsabilidade do Estado em satisfazer as necessidades essenciais da população sob sua guarda, ressaltando-se a obrigação do Estado em relação à saúde dos indivíduos e populações que compõem 
a sociedade que representa, incluindo ações coletivas - promoção da saúde e prevenção da doença - e individuais - cura e reabilitação ${ }^{9}$.

$M$ as, que necessidades essenciais são estas? $\mathrm{Na}$ abordagem adotada aqui, as necessidades essenciais são aquelas que, quando satisfeitas, permitem ao ser humano exercer suas capacidades. Capacidade entendida como a liberdade de uma pessoa poder escolher um modo de vida - 0 que esta pessoa quer ser e fazer -, dentro de um conjunto razoavelmenteamplo de modos devida alternativos ${ }^{7}$. Para tanto, uma pessoa deve ter acesso a um conjunto de meios polivalentes para realizar seus fins - bens e serviços - e condições econômicas e sociais que permitam a esta pessoa converter estes meios em capacidade ou liberdade efetiva para escolher fins e realizá-los - seus modos de vida ${ }^{8}$.

Para N ussbaum ${ }^{5}$, existe um conjunto de capacidades humanas centrais - que marca a pre sença ou ausência da vida humana - e um modo digno ou verdadeiramente humano de exercê las. Entre estas, incluem a capacidade de viver uma vida com longevidade normal, de ter saúde e integridade corporal, de vivenciar emoções, de imaginação e pensamento, de controle sobre 0 ambiente, de preocupar-se com outras espécies, de exercício da razão prática e de afiliação. A razão prática - a capacidade de elaborar e implementar uma concepção do bem ou um plano de vida - e a afiliação - a capacidade viver com e para outras pessoas e a capacidade de ter as bases sociais do auto-respeito - são de especial importância porque moldam e englobam todas as outras, tornando o exercício das mesmas realmente humanas.

A capacidade de ser saudável tem não só um valor em si mesmo, no sentido de que, de modo geral, valorizamos funcionamentos tais como estar bem nutrido, livre de doenças evitáveis e de morte prematura, entreoutros, mas também um valor por ser esta capacidade uma condição ne cessária para o exercício de outras capacidades ${ }^{7,13}$. Como efeito, salienta N ussbaum ${ }^{5}$, uma das condições básicas para o desenvolvimento e exercício de outras capacidades é a presença de um equipamento biológico inato do indivíduo razoavelmentesaudável.

$\mathrm{Na}$ perspectiva que estamos adotando, uma sociedade justa seria aquela em queo Estado efetivamente protege, através de políticas públicas, as capacidades centrais de todos os cidadãos, garantido que elas sejam exercidas acima de um limiar mínimo ${ }^{5}$. Assim sendo, o foco prioritário de preocupação moral do Estado é com as pes- soas que são vulneradas, porque são aquelas que vivem em condições sociais que privam as mesmas do exercício, acima de um limiar mínimo, dessas capacidades ${ }^{12}$. É com estas perspectivas que discutiremos, a seguir, o que é devido a cada um naUTI neonatal, considerando-seajusta alocação dos recursos sanitários públicos.

\section{Proteção e vulneração em UTI s neonatais}

A atenção neonatal cresceu muito no Brasil nos últimos vinte anos, no setor privado e no público, com um excesso deleitos no primeiro eescassez no segundo ${ }^{14,15}$. Este crescimento, no entanto, foi feito sem um adequado planejamento estratégico deambosos setores, não existindo igualdade na distribuição dos leitos de UTI neonatal entre as várias regiões do país, os estados e no interior dos estados ${ }^{14,15}$. Regiões mais desenvolvidas em termos socioeconômicos, como as regiões Sudeste e Sul, concentram os leitos de UTI neonatal, com limitado acesso aos setores da população com menos recursos ${ }^{14,15}$. No estado do Rio deJaneiro, o número de leitos está dentro dos limites recomendáveis, mas com grandeconcentração nas regiões centrais, onde se situa a capital, em detrimento do interior ${ }^{14,16}$. Se tomarmos uma cidade como São Paulo, existe uma alta concentração de leitos nas regiões centrais da cidade e uma baixa concentração nas regiões periféricas, com excesso de leitos no setor privado e escassez no setor público ${ }^{17}$. Por outro lado, não existe um sistema de transporte efetivo para crianças criticamente doentes e mulheres com gravidez de alto risco que permita uma concentração de recursos e experiência em unidades com mais leitos, conforme tendência internacional, favorecendo a criação de pequenas unidades em áreas com pouca densidade populacional ${ }^{14}$. Além disso, existem muitas diferenças na qualidade dos serviços oferecidos, variando de unidades extremamente sofisticadas para outras sem uma estrutura mínima básica ${ }^{14}$.

Um das questões éticas que preocupam diariamente os profissionais de saúde que trabaIham nas UTIs neonatais diz respeito à escolha de quem deveser beneficiado - edeque maneira - com os recursos públicos disponíveis para a atenção neonatal.

As análises realizadas aqui, relativas a esta questão, pressupõem a aceitação do Princípio da Qualidade de Vida, segundo o qual a vida só tem valor se provida de determinadas qualidades ${ }^{18}$. N esta perspectiva, a tomada de decisão sobre 
quem deve ser beneficiado pelas tecnologias mé dicas esta baseada numa avaliação da qualidade de vida que é possível obter com a intervenção médica. Adota-se aqui uma ampla visão de qualidade de vida, em que a decisão sobre o tratamento médico não se baseia, exclusivamente, numa avaliação dos interesses do recém-nascido, mas leva em consideração os interesses da família e da sociedade ${ }^{19}$. No caso da medicina intensiva neonatal, os recém-nascidos não são competentes para avaliar a qualidade de suas próprias vidas e tomar decisões autônomas relativas ao tratamento médico. Pergunta-se então: quem são os que têm autoridade moral para manifestar-se em seu nomee em nome dos interesses aqui em disputa? Se por um lado não parece haver dúvida da necessidade de que os familiares tenham uma participação central no processo, já que são aqueles emocionalmente mais envolvidoseosqueterão quelidar em longo prazo com as conseqüências dessa decisão, o mesmo não ocorre quando pensamos em quem deverá representar os interesses da sociedade nesse de bate. A princípio poderíamos dizer que o ideal seria contarmos com uma "representação" da sociedade em cada unidade hospitalar (ou município ou mesmo estado), para que diretrizes gerais tenham sido estabelecidas ou que assim o sejam em cada caso específico. Esta "representação" estaria dada através dos chamados comitês hospitalares de bioética ou ainda comissões municipais ou estaduais de bioética. N estas comissões, estariam representados diferentes segmentos da sociedade, entre os quais os profissionais de saúde.

Do ponto de vista da teoria das capacidades, levar em consideração a qualidade de vida do recém-nascido significa avaliar, em primeiro lugar, as condições de funcionamento de seu equipamento biológico inato, já que este éa base necessária para que o recém-nascido possa vir a desenvolver e exercer, pelo menos, num nível mínimo, suas capacidades básicas, incluindo funcionamentos tais como ser capaz de viver uma vida de duração considerada razoável, ser capaz demover-se livremente deum lugar para outro e ser capaz de usar a imagi nação e o pensamento e engajar-se em várias formas de interação social ${ }^{5}$. Esse equipamento pode estar detal maneira comprometido que pode prejudicar irreversivelmente a capacidade futura do recém-nascido para 0 exercício desses funcionamentos, entre outros, implicando, para a sua recuperação parcial, altos custos humanos para a criança, a família e a sociedade $^{20}$. Por outro lado, quem tem legitimida- de moral efetiva de avaliar o quanto de incapacidaderepresentará, para cada família ou comunidade, "altos custos humanos"? Será que este é um cálculo meramente técnico, pertinente aos saberes biomédicos? Como afirma Engelhardt $\mathrm{J}^{21}$, "todo o conhecimento médico disponível para guiar a postergação da morte, bem como a prevenção e a amenização do sofrimento, é probabilístico", ou seja, por maior que seja a chance daquele saber refletir uma possível realidade futura, ainda assim não passará de um cálculo probabilístico, sujeito ao imponderável e ao improvável. Além disso, como argumenta $\mathrm{H}_{\text {arris }}^{22}$, se cada pessoa tem direito igual à atenção à saúde, tem também direito igual às suas chances de sobrevida, seja el as quis forem. Segundo este autor, "se aqueles com melhor chance são sempre preteridos, isto inevitavelmente criaria uma aristocracia dos felizardos".

Em uma UTI neonatal, a questão de quem deveser beneficiado - edeque maneira - com os recursos públicos disponíveis deve ser pensada em relação ao três grupos derecém-nascidosque, de forma geral, compõem a demanda pelo atendimento nestes serviços, considerando-se o projeto terapêutico mais adequado - do ponto de vista ético e técnico - para cada recém-nasci$\mathrm{do}^{23,24}$. Destaque se que, no caso de recursos exclusivamente privados e não reembolsáveis mediante políticas de renúncia fiscal (como ocorre em nosso país através do imposto de renda), pode-se pensar em deixar esta decisão exclusivamente à esfera privada dos que podem financiar seus próprios gastos, conforme proposto por Engelhardt J $\mathrm{r}^{18}$, "liberdade e responsabilidade individuais centrada no indivíduo, ou pelo menos na família". Mas a despeito de reconhecermos algumas das dificuldades apontadas por Engel hardt Jr, no caso de países que reconhecem o direito à saúde como um direito fundamental da pessoa humana, como é o Brasil, é preciso que continuemos com a discussão sobre como realizar escolhas racionais capazes de aumentar o grau dejustiça nos dilemas observados na atenção intensiva em neonatos. E retornemos, portanto, à proposta da divisão em três grupos.

0 primeiro grupo - situação com perspectiva - inclui aqueles recém-nascidos que, se o tratamento médico for oferecido, podem sobreviver com qualidade de vida normal ou diminuída, como nas "situações de défi cit físico ou mental, que, todavia, permite uma vida propriamente humana" 25.

Um contingentesignificativo de recém-nascidos deste grupo são prematuros gerados por 
mulheres que não tiveram acesso ao pré-natal cuja condição clínica permitiria quefossem atendidos em unidades assistenciais menos custosas, como são, por exemplo, os berçários intermedi$a^{a}{ }^{0}{ }^{26}$. Cometeríamos uma dupla injustiça senão priorizássemos os recém-nascidos deste grupo, já que uma injustiça foi anteriormente cometida, quando não teve acesso à atenção ao pré-natal.

N este caso, recursos são drenados para as tecnologias de alto custo quando se poderiam gastar menos para evitar que recém-nascidos venham a precisar de atenção intensiva, oferecendo-se um pré natal adequado a todas as gestantes dentro deum sistema de atenção à saúdeintegrado e hierarquizado que contem plasse serviços de medicina intensiva neonatal com graus dife renciados de complexidade para atender recémnascidos com diferentes níveis de gravidade ${ }^{27,28}$.

0 segundo grupo - situação sem esperança situa-se no pólo oposto ao do primeiro, sendo composto por aqueles recém-nascidos portadores decondições clínicas que levam inevitavelmente ao óbito, apesar da intervenção médica - por serem portadores de síndromes incompatíveis com a vida, como a anencefalia, ou extremamenteprematuros - com idadee peso gestacional constantemente sendo alterados em decorrência da progressiva incorporação das biotecnologiasaplicadas à assistência à saúde e que têm como possíveis limites as 23 semanas e os 400 gramas -, entre outras condições clínicas graves em que o equipamento biológico inato do indivíduo está profundamente comprometido e irrecuperável. São recém-nascidos que estão morrendo antes de viverem, isto é, antes de desenvolverem e exercerem, pelo menos num nível mínimo, suas capacidades centrais, vivendo uma vida digna ou verdadeiramente humana. Nestes casos, a morte parece ainda mais terrível e a derrota diante dela mais desconsoladora, sobretudo se considerarmos o grande sofrimento dos familiares, em particular os pais, diante de um filho queamam edepositaram tantas expectativas.

N este contexto, a limitação do esforço terapêutico éatitudeeticamentecorreta, porquemanter artificialmente "as funções vitais sem expectativas razoáveis de recuperação pode prolongar o sofrimento do paciente e seu entorno familiar, chegando a atentar contra a própria dignidade do enfermo" - princípio da não maleficência - , nem sempre representa um equilíbrio favorável entre riscosebenefícios - princípio da beneficência -, implicando uma qualidade de vida muito baixa e pode redundar em excluir pacientes mais necessitados - princípio da justiça ${ }^{29}$.
Desta forma, o projeto terapêutico para este grupo deve estar centrado nos cuidados paliativos, entendidos como o conjunto de medidas dirigidas aos pacientes fora de possibilidades de cura e seus familiares, oferecidos por equipes multiprofissionais, de modo a propiciar uma morte digna para os mesmos ${ }^{30}$. Isto inclui, em relação ao recém-nascido, o alivio da dor e do sofrimento e, em relação aos familiares, o apoio psicológico e espiritual, além de orientação aos pais em relação às próximas gestações, em particular nos casos de malformações congênitas ${ }^{31,30}$.

Nesta abordagem, não se deve transformar 0 direito à vida no direito deimpedir a mortea todo custo, além de um limiteque poderia ser considerado razoável, como se a morte fosse um inimigo que deve ser sempre combatido. Essa postura refletiria "um ideal médico de imortalidade corporal", no qual a "falha em vencer a morte pode ser experimentada como uma falha pessoal", dificultando que os profissionais analisem todas as opções disponíveis, inclusive a morte do paciente, quando 0 tratamento é considerado fútili ${ }^{32}$.

Nos casos de anencefalia, em particular, debate-se, na atualidade, a moralidade do aborto, recorrendo-se a dois argumentos principai ${ }^{33}$. 0 primeiro éo consenso naliteratura médica internacional em relação ao diagnóstico e à impossibilidade da vida extra-uterina do feto, não podendo sua interrupção ser proibida, com base na defesa da vida humana, já que, neste caso, falta ao feto um equipamento biológico inato suficiente saudável, como condição fundamental para que o feto tenha a capacidade ou potencialidade de viver a vida. 0 próprio Conselho Federal de M edicina (CFM ), para efeito detransplante de órgãos e tecidos, considera o anencéfalo um "natimorto cerebral", já que não tem o encéfalo e, portanto, atividade cerebra ${ }^{34}$. 0 segundo argumento é a preocupação com a integridade psíquica da mãe, ressaltando-se o sofrimento para a mesma ao levar a termo gravidez de uma criança que não sobreviverá.

0 terceiro grupo localiza-se entre o primeiro e o segundo grupo, sendo composto por recémnascidos que podem viver, caso o tratamento seja oferecido, mas cuja qualidade de vida futura é extremamente pobre ou questionável - situação sem perspectiva. Algumas destas crianças poderão ser salvas, mas terão que conviver com importantes seqüelas anatômicas e/ou funcionais, algumas vezes acompanhadas de grande sofrimento psíquico, que as impedirão de viver uma vida independente. Sua qualidade de vida futura poderáser mínima ou abaixo do mínimo, envol- 
vendo importante restrição da "capacidade de consciência e de relação com outros seres humanos"25. Contudo, é neste grupo que uma avaliação da qualidade de vida futura do recém-nascido fica mais difícil de ser determinada, incluindo a carga que deve ou pode recair sobre a sociedade e a família, ao investir em um recém-nascido.

No caso de malformações em geral o caso parece ser um pouco menos complexo que no das intercorrências clínicas e cirúrgicas que não apresentam sinais premonitórios. No caso daquelas, deve-se favorecer o exercício da autonomia da mulher, permitindo que a mesma, adequadamente informada, escolha livremente se quer ou não dar à luz a uma criança gravemente deficiente, considerando que as conseqüências desta decisão recaem sobre ela, em primeiro lugar. Nesta perspectiva, exige-se da sociedadeações em duas direções complementares ${ }^{35}$. Por um lado, melhorar continuamente os meios diagnósticos e prognósticos destas malformações congênitas, incluindo-se avaliações dos sofrimentos que poderiam acarretar para a criança, permitindo, desta forma, que a mulher tenha todas as informações pertinentes para uma decisão consciente. Por outro lado, melhorar as condições deacolhimento de pessoas portadoras de necessidades especiais, criando as condições de uma sociedade solidária e viabilizando a decisão do casal de continuar a gravidez sabendo do risco de nascimento de uma criança com estas necessi dades.

Contudo, quanto seria razoável investir em um recém-nascido para que ele possa usufruir, num grau mínimo, das suas capacidades centrais, considerando o mesmo interesse de outros grupos no interior da sociedade?

Esta questão refere-seà distribuição justa dos recursos entre os diferentes coortes de nascimento, envolvendo, por exemplo, a ponderação das necessidades e interesses de coortes formadas por recém-nascidos que sobrevivem com qualidade devida normal ou razoável equesobrevivem com qualidade de vida muito reduzida ${ }^{36,37}$. Assim, por exemplo, em um contexto de escassez de recursos, pode ocorrer que 0 aumento dos recursos para ampliar as chances de estender a vida de recém-nascidos com o equipamento biológico inato significativamente prejudicado até, digamos, uns 40 ou 50 anos, apenas seja possível às custas da diminuição dos recursos distribuídos para recém-nascidos com o equipamento biológico razoavelmente saudável e, portanto, de suas chances de alcançar um ciclo de duração da vida normal (digamos, em torno de 80 ou 90 anos).

Por outro lado, em que grupo incluiríamos os recém-nascidos, nascidos prematuramente ou não, que foram acompanhados através de atendimento pré-natal, mas que desenvolveram uma condição mórbida no período perinatal que os levou a necessitarem dos cuidados passíveis de serem oferecidos em UTIs? Entendemos que se enquadram igualmente no grupo I, cuja característica principal deve ser as capacidades biológicas que persistem nos indivíduos, enão as características do atendimento pré-natal oferecido ou não às mães.

$M$ as, com este enfoque, assegura-se um grau de primaziainaceitável aos aspectos técnicos/biológicos que são, vale sempre repetir, incertos, posto que probabilísticos.

Assim, relativizando-se a pertinência de se conferir apenas à autoridade técnica o poder de decisão, o problema moral que permanece refere-se também a quem tem autoridade moral e baseado em que para determinar a quantidade de recursos que podem/devem ser destinados a uma determinada vida em particular (ainda que não reconhecida pelas autoridades com toda a singularidade que de fato possui) para que possa ser vivida. Ou, perguntando-se de uma outra forma, qual grau de comprometimento é suficiente para que se determine a impropriedade ou inadequação de uma vida determinada, como quando somos chamados a refletir e opinar no contexto de situações clínicas concretas?

\section{Consideraçõesfinais}

Se o conhecimento é probabilístico, a incerteza quanto ao prognóstico nesses casos é ainda mais acentuada, na medida em que existem poucos estudos de follow up que amparem a construção de evidênciasrazoáveis, particularmenteem paísestão desiguais como o Brasil. Daí a necessidadeimperiosa de se conhecer melhor a situação, promovendo-se e estimulando-se a realização desses estudos, de forma que se possa informar e esclarecer de forma mais apropriada as mães e as famílias envolvidas. N ovas eantigas experiências queestão em curso poderiam orientar ou servir como referência para o desenvolvimento deste acompanhamento (por exemplo, nos Estados Unidos ${ }^{38}, \mathrm{Ca}$ nadá39,40 e Europa). A decisão ética será também mais qualificada quanto melhor for a informação clínica queestiver disponível, inclusivesobre prognósticos. Lembrando sempre, entretanto, quenesses tempos de medicina baseada em evidências e de glamourização da racionalização, esta é uma permanentefontedeilusões. Estes dois documen- 
tos canadenses, acima referidos, fazem questão de lembrar-nos disso, ao destacarem que as recomendações propostas não devem indicar um curso único para o tratamento, buscando-se variações que possam ser mais adequadas às circunstâncias individuais. Assim, como afirmou Suzan-

\section{Colaboradores}

CDM Ribei ro teve a concepção inicial do artigo e realizou a primeira versão do texto. $S$ Rego discutiu a versão inicial do artigo e contribuiu de forma suficiente para assumir a co-autoria do artigo na concepção e redação da versão final do texto.
neToce ${ }^{41}$, "decisões sobretratamento ereanimação apropriadas de lactentes de baixo-peso extremo não podem ser nunca nem o triunfo da esperança sobre a razão nem a vitória do ego sobre a incerteza". E, especialmente, não deve nunca deixar de envolver os pais da criança nesta decisão.

\section{Referências}

1. Rawls J. U ma teoria da justiça. São Paulo: Martins Fontes; 1997.

2. Rawls J. O liberalismo político. $2^{\mathrm{a}}$ ed. São Paulo: Ática; 2000.

3. Engelhardt J r HT. Fundamentos da bioética. São PauIo: Edições Loyola; 1998.

4. Society of Critical Care M edicine (SCCM). Definitions, 1999 [acessado 2001 jun 1]. Disponível em: http://www.sccm.org/accm/guidelines/ guide_body_definitions.httm

5. Nussbaum $\bar{M} C$. In defense of universal values. In: Nussbaum MC, editor. Women and human development. The capabilities approach. Cambridge: Cambridge University Press; 2000. p 34-110.

6. Nussbaum MC. Beyond the social contract: toward global justice. The Tanner Lectures on Human Values. Cambridge: Cambridge University; 2003.

7. Sen AK. Desenvolvimento como liberdade. São PauIo: Companhia das Letras; 2000.

8. Sen AK. Desigualdade reexaminada. Rio de Janeiro: Record; 2001.

9. Schramm FR, Kottow M. Principios bioéticos en salud pública: limitaciones y propuestas. Cad Saúde Pública 2001; 17(4):949-956.

10. Kottow $\mathrm{MH}$. Vulnerability: what kind of principle is it? M ed H ealth Care Philos 2004; 7:281-287.

11. Kottow M. The vulnerable and the susceptible. Bioethics 2003; 17(5-6):461-471.

12. Ribeiro CDM, Schramm FR. Atenção médica, transplante de órgãos e tecidos e políticas de focalização. Cad Saúde Pública 2006; 22:1945-1953.

13. Sen $A K$. Por qué la equidad en salud. Rev Panam Salud Publica 2001; 11(5-6):302-309.

14. Barbosa AP. Neonatal and pediatric intensive care in Brazil: the ideal, the real, and the possible. J Pediatr (Rio J) 2004; 80(6):437-438.

15. Kamada I, Rocha SM M, Barbeira CBS. Internações em Unidades de Terapia Intensiva Neonatal no Brasil - 1998 - 2001. Rev Lat Am Enfermagem 2003; 11(4):436-443.

16. Barbosa AP, Cunha JLA, Carvalho ERM, Portella AF, Andrade M PF e Barbosa M CM. Terapia Intensiva Neonatal e pediátrica no Rio de Janeiro: distribuição de leitos e análise de equidade. Rev Assoc M ed Bras 2002; 48(4):303-311.

17. 1Souza DC, Troster EJ, Carvalho WB, Shin SH, Cordeiro AMG. Availability of pediatric and neonatal intensive care units in city of São Paulo. J Pediatr (Rio J) 2004; 8(6):453-460. 
18. M ori M . A bioética: sua natureza e história. Humanidades 1994; 9(4):333-341.

19. Norup M. Limits of neonatal treatment: a survey of attitudes in the Danish population. J Med Ethics 1998; 24:200-206.

20. Campbell AGM, M CH affie HE. Prolonging life and allowing death: infants. J Med Ethics 1995; 21:339344.

21. Engelhardt Jr HT. A bioética do risco: enfrentando a finitude humana. In: Schramm FR, Rego S, Braz M, Palácio $M$, organizadores. Bioética, risco e proteção. Rio de Janeiro: UFRJ/FIOCRUZ; 2005. p. 133-147.

22. Harris J. What is the good of health care? Bioethics 1996; 10(4):269-291.

23. M CH affie HE, Cuttini GB, Randag L, M ousty R, Duguet AM, Wennergren B, Benciolini P. Withholding/withdrawing treatment from neonates: legislation and official guidelines across Europe. J M ed Ethics 1999; 25:440-446.

24. Ribeiro CDM . "Cultura dos limites", justiça. sanitária e alocação de recursos: argumentações racionais acerca dos conflitos morais em medicina intensiva neonatal, do ponto de vista dos profissionais intensivistas [tese]. Rio de Janeiro (RJ): Escola Nacional de Saúde Pública, Fundação Oswaldo Cruz; 2003.

25. Sanchez-Gonzalez, M A. La calidad de vida en la toma de decisiones sobre enfermos terminales. Quaderni di Bioetica e Cultura 1995; 3:87-101.

26. Ribeiro CDM. Bioética e neonatologia. In: Schramm FR, Braz M, organizadores. Bioética e Saúde. Novos Tempos para mulheres e crianças? Rio de Janeiro: Fiocruz; 2005. p. 219-236.

27. Goodman DC, Fisher ES, Little GA, Stukel TA, Chang C. Are neonatal intensive care resource located according to need? Regional variation in neonatologists, bed, and low birth weight newborns. Pediatrics 2001; 108(2):426-431.

28. Viguri NPR, Dominique NP. Consideraciones sobre microasignación y macroasignación de los recursos sanitarios. In: Rubi JAG, Campos RA, coordenadores. Bioética y medicina intensiva: dilemas éticos en el paciente crítico. Barcelona: Edika M ed/SEM ICYUC; 1998. p. 153-179.

29. Rubi JAG, Navarro AR. Limitación del esfuerzo terapêutico. Fundamentos éticos. In: Rubi JAG Campos RA, coordenadores. Bioética y medicina intensiva: dilemas éticos en el paciente crítico. Barcelona: Edika M ed/SEMICYUC; 1998. p. 73-78.

30. Schramm FR. Cuidados paliativos: aspectos filosóficos. Rev Bras Cancerol 1999; 45(4):57-63.

31. American Academy of Pediatrics. Palliative care for children. Pediatrics 2000; 106 (2):351-357.
32. Marsden $\mathrm{C}$. An ethical assessment of intensive care Int J Technol Assess Health Care 1992; 8(3):408-418.

33. Diniz D. Aborto e inviabilidade fetal: el debate brasileño. Cad Saúde Pública 2005; 21(2):634-639.

34. Conselho Federal de Medicina. Resolução nº 1.752. Autorização ética do uso de órgãos e/ou tecidos de anencéfalo para transplante, mediante autorização prévia dos pais. Brasília: Conselho Federal de Medicina; 2004.

35. Barchifontaine CP. Nascer ou não com graves deficiências congênitas? In: Garrafa V, Pessini L, organizadores. Bioética: poder e injustiça. São Paulo: Loyola; 2003. p. 243-254.

36. Daniels N. Health-care needs and distributive justice. In: Daniels $\mathrm{N}$, editor. Justice and justification: reflexive equilibrium in theory and practice. New York: Cambridge University Press; 1996. pp. 179-207.

37. Ribeiro CDM, Schramm FR. A necessária frugalidade dos idosos. Cad Saúde Pública 2004; 20(5):11411159.

38. MacD onald $\mathrm{H}$, Committee on Fetus and Newborn. Perinatal Care at the Threshold of Viability. Pediatrics [periódico na Internet] 2002 [acessado em 2006 nov 2]; 110:[cerca de 4 p.]. Disponível em: http:// www.pediatrics.org/cgi/content/full/110/5/1024

39. Society of Obstetricians and Gynaecologists of Canadá - M aternal-Fetal M edicine Committee \& Canadian Paediatric Society - Fetus and Newborn Committee. Management of the woman with threatened birth of an infant of extremely low gestational age. Reference $\mathrm{N}^{\circ}$. FN 94-01. Can M ed Assoc] [periódico na Internet] 1994 [acessado 2006 nov 2]; 151(5):[cerca de 5 p.]. Disponível em: http:// www.cps.ca/english/statements/FN/fn94-01.htm

40. Canadian Paediatric Society - Fetus and Newborn Committee. Levels of neonatal care Position Statement (FN 2006-02). Paediatr Child Health 2006; 11(5):303-306.

41. Toce S. Ethical Decision Making In the Delivery Room [acessado 2006 nov 2]. Disponível em: http:/ / www.cardinalglennon.com/internet/home/ net10hom.nsf/0/d7f99e5d7c4cb5d586256c62006c $\mathrm{db12}$ ?O penD ocument\#top\#top

Artigo apresentado em 20/03/2007

Aprovado em 12/12/2007

Versão final apresentada em 15/01/2008 